\title{
Effects of Helicobacter pylori eradication on the natural history of lymphocytic gastritis
}

\author{
M Hayat, D S Arora, M F Dixon, B Clark, S O'Mahony
}

\begin{abstract}
Background-Lymphocytic gastritis is characterised by an accumulation of $1 y m-$ phocytes in the surface epithelium of the stomach. Lymphocytic gastritis has been linked to coeliac disease and Helicobacter pylori infection.
\end{abstract}

Aims-To determine whether $H$ pylori eradication leads to resolution of the lymphocytic infiltrate and clinical improvement in patients with lymphocytic gastritis, and to determine their HLA status.

Methods-The Leeds Dyspepsia Questionnaire (LDQ) was administered to 13 patients with lymphocytic gastritis. $H$ pylori serology, ${ }^{13} \mathrm{C}$ urea breath test (UBT), and upper gastrointestinal endoscopy with sampling of the duodenum, antrum, and corpus were done in all cases and the HLA status was determined. Eleven patients had at least one positive test for $H$ pylori. Patients with lymphocytic gastritis and $H$ pylori infection were treated with a one week course of omeprazole, clarithromycin, and metronidazole. Gastric and duodenal intraepithelial lymphocyte (IEL) counts were performed, along with histological assessment of gastric and duodenal biopsies before and after $\boldsymbol{H}$ pylori eradication.

Results-Two months after treatment there was a significant reduction in gastric IEL counts in both antrum and corpus. There was no significant change in duodenal IEL counts before and after eradication. According to the Sydney grading there was significant improvement in corpus inflammation after eradication. The patients histologically $H$ pylori positive before treatment became $H$ pylori negative. Dyspepsia scores also improved significantly after treatment.

Conclusions-H pylori eradication treatment in patients with lymphocytic gastritis causes significant improvement in the gastric IEL infiltrate, corpus inflammation, and dyspeptic symptoms. $H$ pylori serology is frequently positive when histology and UBT are negative. Lymphocytic gastritis may represent a specific immune response to $H$ pylori infection. (Gut 1999;45:495-498)

Keywords: lymphocytic gastritis; intraepithelial lymphocytes; Helicobacter pylori; HLA status; coeliac disease

Lymphocytic gastritis is characterised by an accumulation of lymphocytes in the surface and foveolar epithelium of the stomach. It is found in approximately $1 \%$ of gastric biopsy specimens from dyspeptic patients. ${ }^{12}$ The diagnostic threshold for lymphocytic gastritis is usually taken as greater than 25 intraepithelial lymphocytes (IELs) per 100 epithelial cells. ${ }^{13}$ This special type of gastritis may be associated with a normal appearing gastric mucosa. However, the classical endoscopic appearance comprises prominent rugal folds bearing small nodular elevations surmounted by small grey-white erosions with hyperaemic margins, maximal in the body and fundus. ${ }^{13}$ This endoscopic picture is generally termed "varioliform" gastritis.

The aetiology of lymphocytic gastritis is unknown. It has been attributed to an atypical response to Helicobacter pylori infection. ${ }^{1} \mathrm{Al}-$ though many patients with lymphocytic gastritis are seropositive for $H$ pylori, its presence is not usually confirmed histologically. Lymphocytic gastritis is frequently found in patients with coeliac disease. In one study of 22 patients with coeliac disease, $50 \%$ had lymphocytic gastritis. ${ }^{4}$ The IELs in the stomach and small bowel were positive for MT-1, indicative of T cell infiltration. It has also been suggested that lymphocytic gastritis may represent part of a diffuse lymphocytic gastroenteropathy which varies in its expression from site to site. ${ }^{5}$

Gut intraepithelial lymphocytes are almost entirely CD3 + T cells, and approximately $80 \%$ of them are CD8+. ${ }^{67}$ In humans up to $15 \%$ of IELs carry the $\mathrm{T}$ cell receptor, which is found on less than 3\% of peripheral blood lymphocytes. ${ }^{6} 7$

We investigated the effects of $H$ pylori eradication on the natural history of lymphocytic gastritis and also determined the HLA status of our patients with lymphocytic gastritis in an attempt to explore further the relation of lymphocytic gastritis with coeliac disease.

\section{Methods}

Thirteen newly diagnosed patients with a histological diagnosis of lymphocytic gastritis were identified and were invited to take part in the study. Table 1 presents their clinical details. Informed consent was obtained from all patients. Prior to endoscopy the Leeds Dyspepsia Questionnaire, a validated symptom questionnaire, ${ }^{8}$ was performed. Venous blood was taken for $H$ pylori serology and HLA status. A carbon-13 urea breath test was performed. At endoscopy, two biopsy specimens were taken from the second part of the duodenum, gastric antrum, and body using standard biopsy forceps.

Abbreviations used in this paper: IEL, intraepithelial lymphocyte; PBMC, peripheral blood mononuclear cell; UBT, urea breath test. 
Table 1 Clinical details of patients with lymphocytic gastritis

\begin{tabular}{lllll}
\hline Patient no & Age $(y)$ & Sex & OGD & $\begin{array}{l}\text { Presenting } \\
\text { symptoms }\end{array}$ \\
\hline 1 & 75 & $\mathrm{M}$ & $\mathrm{N}$ & IDA \\
2 & 79 & $\mathrm{~F}$ & $\mathrm{~N}$ & Dyspepsia \\
3 & 61 & $\mathrm{M}$ & $\mathrm{AE}$ & Dyspepsia \\
4 & 36 & $\mathrm{~F}$ & $\mathrm{~N}$ & Diarrhoea \\
5 & 50 & $\mathrm{M}$ & $\mathrm{PHG} / \mathrm{OV} /$ & $\mathrm{IDA}$ \\
& & & $\mathrm{EG}$ & \\
6 & 53 & $\mathrm{M}$ & $\mathrm{N}$ & Dyspepsia \\
7 & 65 & $\mathrm{~F}$ & $\mathrm{VG}$ & Dyspepsia \\
8 & 52 & $\mathrm{~F}$ & $\mathrm{GE} / \mathrm{EG}$ & IDA \\
9 & 80 & $\mathrm{M}$ & $\mathrm{GU}$ & IDA \\
10 & 20 & $\mathrm{~F}$ & $\mathrm{~N}$ & Dyspepsia \\
11 & 23 & $\mathrm{M}$ & $\mathrm{VG}$ & Dyspepsia \\
12 & 53 & $\mathrm{M}$ & $\mathrm{GE}$ & Diarrhoea \\
13 & 70 & $\mathrm{~F}$ & $\mathrm{D}$ & Dyspepsia \\
\hline
\end{tabular}

$\mathrm{AE}$, antral erythema; $\mathrm{D}$, duodenitis; $\mathrm{EG}$, erythematous gastritis GE, gastric erosions; GU, gastric ulcer; IDA, iron deficiency anaemia; N, normal; OGD, oesophagogastroduodenoscopy; $\mathrm{OV}$, oesophageal varices; $\mathrm{PHG}$, portal hypertensive gastropathy; VG, varioliform gastritis.

HISTOLOGY

Specimens were fixed in 10\% buffered formalin, embedded in paraffin wax, and sections stained with haematoxylin and eosin (H\&E). A modified Giemsa stain was used to detect $H$ pylori. The H\&E stained sections were examined at $\times 400$ magnification and the number of IELs and epithelial cell nuclei in an uninterrupted length of surface and foveolar epithelium were counted. Based on previous studies, $^{35}$ the ratio of 25 IELs: 100 epithelial cells was used as a limit for inclusion into this study. Gastritis was classified according to the updated Sydney System. ${ }^{9}$

\section{IMMUNOHISTOLOGY}

Duodenal and gastric biopsy specimens were also immunostained using a three step immunoperoxidase technique with anti-CD3 (pan $\mathrm{T}$ cell) monoclonal antibodies (from Dako, High Wycombe, UK) and IEL counts were performed.

IMMUNOSTAINING

The ABC technique was employed for immunostaining using the Duet kit (Dako Ltd, Denmark). The paraffin wax sections were pretreated by pressure cooking in citrate buffer at $\mathrm{pH} 6$ for 90 seconds at full pressure. The polyclonal $H$ pylori antibody was used at a dilution of $1 / 100$.

\section{HLA STATUS}

$D N A$ isolation

Genomic DNA was obtained from peripheral blood mononuclear cells (PBMC) according to the method of Miller et al. ${ }^{10}$ Extracted DNA was redissolved in TE buffer, adjusted to 1 $\mu \mathrm{g} / \mu \mathrm{l}$ by ultraviolet spectrophotometry and stored at $4^{\circ} \mathrm{C}$ until use.

HLA genotyping

PBMC DNA samples were typed for HLADRB and DQB antigens using a 40 reaction PCR-SSP system with amplification primers as described by Bunce et al. ${ }^{11}$ PCR product was electrophoresed in a $1.5 \%$ agarose gel containing $0.5 \mu \mathrm{g} / \mathrm{ml}$ ethidium bromide and visualised using ultraviolet illumination.
H PYLORI SEROLOGY

Serum samples from all 13 subjects were assayed for $H$ pylori IgG antibodies (Sigma Diagnostics). H pylori IgG antibody titres were also assayed by Premier $H$ pylori kit (supplied by Launch Diagnostics, UK), before and after $H$ pylori eradication in seven patients (patients 2, 4-9; table 2).

Patients were classed as $H$ pylori positive on the basis of at least one positive test. Previous study from our unit ${ }^{5}$ has shown that in patients with lymphocytic gastritis $H$ pylori serology is frequently positive when the histology is negative. Past studies have also suggested that lymphocytic gastritis could be a manifestation of an atypical host immune response to $\mathrm{H}$ pylori. ${ }^{1}$ We therefore classed our patients as $H$ pylori positive on the basis of positive serology even when the histology and/or the ${ }^{13} \mathrm{C}$ urea breath test was negative. On this basis patients with lymphocytic gastritis and $H$ pylori infection were treated with a standard one week triple therapy course of omeprazole $20 \mathrm{mg}$ once daily, clarithromycin $250 \mathrm{mg}$ twice daily, and metronidazole $400 \mathrm{mg}$ twice daily. After an interval of two months, the Dyspepsia Questionnaire, endoscopy with duodenal and gastric biopsies, and ${ }^{13} \mathrm{C}$ urea breath test were repeated. In seven patients (patients 2, 4-9) who received $H$ pylori eradication, $H$ pylori serology was repeated after an interval of one year to document a fall in antibody titre to $H$ pylori. Gastric and duodenal IEL counts were performed along with Sydney grading ${ }^{9}$ of gastric biopsy specimens and histological assessment for chronic inflammation and degree of villous atrophy of duodenal biopsy specimens, before and after $H$ pylori eradication by an experienced pathologist (MFD) who was blinded to any clinical data. CD3 counts on gastric and duodenal biopsy specimens were performed by a second pathologist (DSA). The study was approved by the local research ethics committee.

\section{STATISTICAL ANALYSIS}

For comparison of paired non-parametric data the Wilcoxon sign rank test was used. To assess the correlation of CD3 and H\&E lymphocyte counts, Pearson's test was used. A p value less than 0.05 was regarded as significant.

Table 2 Laboratory findings in patients with lymphocytic gastritis

\begin{tabular}{lllll}
\hline Patient no & $\begin{array}{l}\text { Hpylori } \\
\text { histology }\end{array}$ & $\begin{array}{l}\text { H pylori } \\
\text { serology }\end{array}$ & ${ }^{13}$ C UBT & $\begin{array}{l}\text { D2 } \\
\text { histology }\end{array}$ \\
\hline 1 & - & + & - & IEL \\
2 & - & + & + & N \\
3 & + & + & - & N \\
4 & - & + & - & IEL \\
5 & - & + & - & N \\
6 & - & + & - & VA \\
7 & + & + & + & VA \\
8 & - & + & - & N \\
9 & - & + & - & N \\
10 & - & + & - & IEL \\
11 & + & + & - & IEL \\
12 & - & - & - & N \\
13 & - & - & - & N
\end{tabular}

D2, second part of the duodenum; IEL, intraepithelial lymphocytes; N, normal; VA, villous atrophy. 
Table 3 Helicobacter pylori titres pre- and post-H pylori eradication in seven patients

\begin{tabular}{lll}
\hline Patient no & Pre-eradication titres & Post-eradication titres \\
\hline 1 & 15.50 & 9.28 \\
2 & 1.48 & 8.88 \\
3 & 6.18 & 1.04 \\
4 & 5.23 & 0.05 \\
5 & $>11.24$ & 7.37 \\
6 & 7.89 & 6.09 \\
7 & 7.69 & 8.78 \\
\hline
\end{tabular}

\section{Results}

ENDOSCOPY

Of 13 patients, endoscopy was normal in five. In two patients endoscopic appearance was that of varioliform gastritis. One endoscopy showed erythema of the antrum. Two patients had erythematous gastritis, one had gastric erosions, one had duodenitis, and one had a small gastric ulcer (table 1).

One patient (no 5) also had portal hypertensive gastropathy and early oesophageal varices secondary to chronic ethanolic liver disease.

H PYLORI HISTOLOGY AND SEROLOGY

Of 13 patients with lymphocytic gastritis, 11 had at least one positive test for $H$ pylori (table 2). All 11 were seropositive for $H$ pylori. In three of $11, H$ pylori was found on histology; one of these also had a positive ${ }^{13} \mathrm{C}$ urea breath test and one other had a positive ${ }^{13} \mathrm{C}$ urea breath test. Two patients (nos 10,11) with lymphocytic gastritis who were $H$ pylori positive were lost to follow up and were excluded from the analysis. We measured paired pre- and posteradication IgG antibody titres in seven patients. Titres fell in five and rose in two (table 3). Intraepithelial lymphocyte counts were performed in nine patients (nos 1-9) before and after $H$ pylori eradication. In seven of these, lymphocytic gastritis was corpus predominant.

On H\&E stained sections the median IEL count in the antrum was 17.0 per 100 epithelial cells (range 7.0-74.0) and 6.0 (range 1.0-19.0) before and after $H$ pylori eradication respectively. Gastric body IEL counts were performed on seven patients as in two patients (nos 2, 3) the pre-eradication biopsies were all of antral type mucosa. The median IEL count in the corpus pre- $H$ pylori eradication was 44.0 (range 28.0-81.0) and posteradication was 10.0 (range 3.0-30.0). There was a significant reduction in the IEL count in both antrum $(\mathrm{p}=0.003$; fig 1$)$ and corpus $(\mathrm{p}=0.015$; fig 2$)$ after $H$ pylori eradication treatment.

The median IEL count in the duodenum was 16.0 (range 7.0-71.0) and 10.0 (range 6.0-67.0) pre- and post- $H$ pylori eradication respectively, and although there was a trend towards a fall in the duodenal IEL count after $H$ pylori eradication, it was not statistically significant. On sections stained with anti-CD3 monoclonal antibodies the median IEL count in the antrum was 11.6 (range 3.6-34.4) and 2.7 (range $0.19-9.2$ ) before and after $H$ pylori eradication respectively, and median corpus counts were 30.35 (range 19.1-72.7) pre- $H$ pylori eradication and 5.3 (range 1.14-25.7) post- $H$ pylori eradication.

There was a significant reduction in the CD3 and IEL counts in both antrum $(\mathrm{p}=0.046)$ and

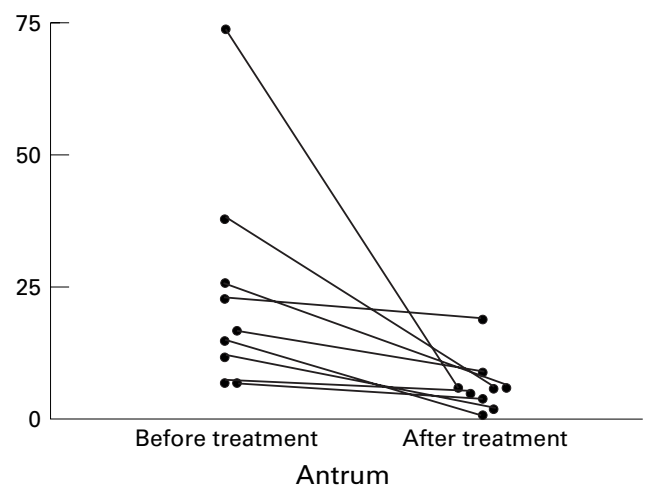

Figure 1 Effect of $H$ pylori eradication on antral intraepithelial lymphocytes.

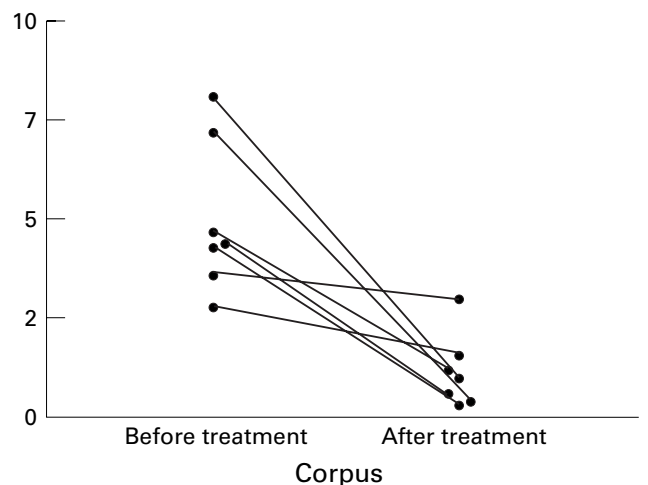

Figure 2 Effect of $H$ pylori eradiation on corpus intraepithelial lymphocytes.

corpus $(\mathrm{p}=0.007)$ post $-H$ pylori eradication. There was also a significant correlation $(p<0.0001$, coefficient $=0.852$ (two tailed) $)$ between the H\&E and CD3 IEL counts. However $\mathrm{H} \& \mathrm{E}$ counts were almost invariably higher than CD3 counts, the mean difference being 7.48. Duodenal (second part) histology was normal in $7 / 13$ patients. Two patients had mild villous atrophy, while four had increased IELs with a normal villous architecture. The two patients with mild villous atrophy were not commenced on a gluten free diet, as the degree of villous atrophy was thought to be insufficient to diagnose coeliac disease. Sydney grading of the gastric biopsy specimens before and after $H$ pylori eradication revealed a significant improvement in the corpus inflammation $(\mathrm{p}<0.03)$ after $H$ pylori eradication. Patients $H$ pylori positive on histology and ${ }^{13} \mathrm{C}$ UBT, turned negative posteradication.

IMMUNOSTAINING

Eighteen pre- and post-treatment biopsy specimens (patient nos 1-9) were immunostained by the technique as described above. The slides were examined "blind" to previous histological results. Two cases (nos 3,7) were designated positive; both were pretreatment biopsies.

DYSPEPSIA SCORES

Dyspepsia scores in the patients receiving $H$ pylori eradication therapy, as assessed by the Leeds Dyspepsia Questionnaire were also significantly improved posteradication $(\mathrm{p}<0.03)$. The median dyspepsia scores were 
4.0 (range $0-6.0$ ) and 0 , pre- and posteradication respectively.

HLA STATUS

HLA status was available on $10 / 13$ patients; seven of the $10(70 \%$; nos $1,3,4,5,7,8,11)$ possessed the DQ2 molecule encoded by the allele $\mathrm{DQB} 1{ }^{\star} 0201$ as compared with $24 \%$ of the controls, a relative risk of $3 \%$. DQB $1{ }^{\star} 0201$ is possessed by $95 \%$ of coeliac patients compared with $20-30 \%$ of controls. ${ }^{12}{ }^{13}$ The control population comprised 100 "normal" individuals (volunteer donors), randomly selected from the local population, and were tissue typed by the same methods as the study group.

\section{Discussion}

Previous studies have suggested that lymphocytic gastritis could be a manifestation of an atypical host immune response to $H$ pylori. ${ }^{14}$ In our study $11 / 13$ patients with lymphocytic gastritis were $H$ pylori positive; we have shown histological and symptomatic improvement in 9/11 of these patients following $H$ pylori eradication, which lends some support to this hypothesis. The relatively low prevalence of the organism on histology, ${ }^{15}$ and the discrepancy between the histological and serological $H$ pylori status in patients with lymphocytic gastritis is in keeping with previous studies. ${ }^{15}$ Thus despite positive serology in all these patients, even the "gold standard" histological test has failed to reveal Helicobacter organisms, either bacterial or coccoid. Given the beneficial effect of eradication treatment we assume that bacteria are present in very low numbers and are not represented in the histological sections.

We have also found that the histological expression of lymphocytic gastritis is variable throughout the stomach and duodenum, as shown in previous studies, ${ }^{13}$ and confirm that the endoscopic appearances in lymphocytic gastritis can vary from normal to the characteristic varioliform gastritis picture. In this study six patients with lymphocytic gastritis had variable degrees of histological abnormality in the duodenum, consistent with a previous study. ${ }^{5}$ Two patients had mild patchy villous atrophy, while the other four patients had increased IEL but with normal villous architecture. Increased small intestinal IEL counts are present in patients with treated coeliac disease following gluten challenge, ${ }^{16}$ dermatitis herpetiformis, ${ }^{17}$ first degree relatives of patients with coeliac disease, ${ }^{18}$ and those with low grade small intestinal enteropathy. ${ }^{19}$ The finding of these histological abnormalities in the duodenal mucosa gives strength to the hypothesis that at least some patients with lymphocytic gastritis have a more generalised disturbance of mucosal immunity, predisposing them to gluten related enteropathy.

We have also shown that the majority of patients with lymphocytic gastritis possess the
DQ2 molecule encoded by the allele DQB $1^{\star} 0201$ which is also possessed by $95 \%$ of the patients with coeliac disease. These findings tentatively suggest a strong association of lymphocytic gastritis with coeliac disease, and lymphocytic gastritis may be a genetically restricted, specific immune response to $H$ pylori infection.

In summary, we have shown that in noncoeliac patients with lymphocytic gastritis who have at least one positive test for $H$ pylori, eradication treatment results in both histological and clinical improvement.

We wish to thank Dr Mark Wilcox, Consultant Microbiologist, for providing the $H$ pylori serology data, and Miss Julie Mackintosh for preparing the manuscript.

1 Dixon MF, Wyatt JI, Burke DA, et al. Lymphocytic gastritis: relationship to Campylobacter pylori infection. I Pathol 1988;154:125-32.

2 Jaskiewicz K, Price SK, Zak J, et al. Lymphocytic gastritis in non-ulcer dyspepsia. Dig Dis Sci 1991;36:1079-83.

3 Haot J, Hamichi L, Wallez L, et al. Lymphocytic gastritis: a newly described entity: a retrospective endoscopic and histological study. Gut 1988;29:1258-64.

4 Wolber R, Owen D, del Buono L, et al. Lymphocytic gastritis in patients with celiac sprue or sprue like intestinal disease. Gastroenterology 1990;98:310-15.

5 Lynch DAF, Sobala GM, Dixon MF, et al. Lymphocytic gastritis and associated small bowel disease: a diffuse lymphocytic gastroenteropathy? F Clin Pathol 1995;48:939-45.

6 Abreu Martin MT, Targan SR. Regulation of immune responses of the intestinal mucosa. Crit Rev Immunol 1996; 16:277-309.

7 Lynch S, Kelleher D, Feighery C, et al. Flow cytometric analysis of intraepithelial lymphocytes from human small intestinal biopsies reveals populations of CD4+CD8+ and CD80 alpha alpha+ cells. Eur $\mathcal{F}$ Gastroenterol Hepatol 1993; 5:907-12.

8 Moayyedi P, Duffett S, Braunholtz D, et al. Leeds Dyspepsia Questionnaire; a valid tool for measuring the presence and severity of dyspepsia. Aliment Pharmacol Ther 1998;12: $1257-62$.

9 Dixon MF, Genta RM, Yardley JH, et al. Classification and grading of gastritis. The updated Sydney System. InterHouston, 1994. Am f Surg Pathol 1996;20:1161-81.

10 Miller SA, Dykes DD, Polesky HS, et al. A simple salting out procedure for extracting DNA from human nucleotide cells. Nucleic Acids Res 1988;16:1215.

11 Bunce M, O'Neill CM, Barnardo MG, et al. Phototyping comprehensive DNA typing for HLA-A, B, C, DRB1, DRB3, DRB4, DRB5 and DQB1 by PCR with 144 primer mixes utilising sequence-specific primers (PCR-SSP). Tissue Antigens 1995;46:355-67.

12 Tosi R, Vismara D, Tanigaki N, et al. Evidence that celiac disease is primarily associated with a DC locus allelic specificity. Clin Immunol Immunopathol 1983;28:395-404.

13 Corazza GR, Trabacchi P, Frissoni M, et al. DR and non-DR1a allotypes are associated with susceptibility to coeliac disease. Gut 1985;26:1210-13.

14 Niemela S, Karttunen T, Kerola T, et al. Ten year follow up study of lymphocytic gastritis: further evidence on Helicobacter pylori as a cause of lymphocytic gastritis and corpus gastritis. F Clin Pathol 1995;48:1111-16.

15 Jones EA, Flejou J-F, Potet F, et al. Lymphocytic gastritis: a clinicopathological study of 32 patients. Eur $\mathcal{F}$ Gastroenterol Hepatol 1990;2:367-72.

16 Marsh MN. Studies of intestinal lymphoid tissue. XI-The immunopathology of cell-mediated reactions in gluten sensitivity and other enteropathies. Scanning Microsc 1988;2: 1663-84.

17 Gawkrodger DJ, McDonald C, O'Mahony S, et al. Small intestinal function and dietary status in dermatitis herpetiformis. Gut 1991;32:377-82.

18 Marsh MN, Bjarnason I, Shaw J, et al. Studies of intestinal lymphoid tissue. XIV-HLA status, mucosal morphology, permeability and epithelial lymphocyte populations in first degree relatives of patients with coeliac disease. Gut 1990; 31:32-6.

19 Marsh MN. Gluten, major histocompatibility complex, and the small intestine. A molecular and immunologic approach to the spectrum of gluten sensitivity ("celiac sprue"). Gastroenterology 1992;102:330-54. 